\title{
Favourable effects of fenofibrate on lipids and cardiovascular disease in women with type 2 diabetes: results from the Fenofibrate Intervention and Event Lowering in Diabetes (FIELD) study
}

\author{
Michael C. d'Emden • Alicia J. Jenkins • Liping Li • Diana Zannino • Kristy P. Mann • \\ James D. Best • Bronwyn G. A. Stuckey • Kris Park • Juha Saltevo • Anthony C. Keech • \\ on behalf of the FIELD Study Investigators
}

Received: 19 January 2014 / Accepted: 3 July 2014 /Published online: 23 August 2014

(C) Springer-Verlag Berlin Heidelberg 2014

\begin{abstract}
Aims/hypothesis In the double-blind placebo-controlled Fenofibrate Intervention and Event Lowering in Diabetes trial $(n=9,795)$, fenofibrate reduced major cardiovascular events in type 2 diabetes. Sex-related differences in fenofibrate response could be clinically relevant and were pre-specified analyses.
\end{abstract}

Electronic supplementary material The online version of this article (doi:10.1007/s00125-014-3344-3) contains peer-reviewed but unedited supplementary material, which is available to authorised users.

\section{C. d'Emden}

Endocrine Research Unit, Royal Brisbane Hospital,

Brisbane, QLD, Australia

\section{C. d'Emden}

Department of Medicine, University of Queensland,

Brisbane, QLD, Australia

$\cdot$ L. Li $\cdot$ D. Zannino $\cdot$ K. P. Mann $\cdot$ A. C. Keech $(\square)$

National Health and Medical Research Council Clinical Trials Centre, University of Sydney, Locked Bag 77, Camperdown, Sydney, NSW 1450, Australia

e-mail: fieldtrial@ctc.usyd.edu.au

\section{A. J. Jenkins $\cdot$ J. D. Best}

Department of Medicine, University of Melbourne,

Melbourne, VIC, Australia

B. G. A. Stuckey

Keogh Institute for Medical Research and Sir Charles Gairdner

Hospital, Perth, WA, Australia

K. Park

Nepean Hospital, Sydney, NSW, Australia

J. Saltevo

Department of Medicine, Central Finland Central Hospital,

Jyväskylä, Finland
Methods Women $(n=3,657)$ and men $(n=6,138)$ with type 2 diabetes not using statins were assigned fenofibrate ( $200 \mathrm{mg} /$ day) or placebo for 5 years. Effects on lipoproteins and total cardiovascular events were evaluated by sex.

Results Baseline total, LDL-, HDL- and non-HDL cholesterol and apolipoproteins A-I and B differed between sexes, and these and triacylglycerol levels improved with fenofibrate in both sexes (all $p<0.001$ ). Fenofibrate reduced total, LDL- and non-HDL cholesterol and apolipoprotein B more in women (all $p<0.001$ ), independent of menopausal status and statin uptake. Adjusted for covariates, fenofibrate reduced total cardiovascular outcomes (cardiovascular death, fatal and non-fatal stroke and carotid and coronary revascularisation) by $30 \%$ in women $(95 \%$ CI $8 \%, 46 \% ; p=0.008)$ and $13 \%$ in men $(95 \% \mathrm{CI}-1 \%$, $24 \% ; p=0.07)$ with no treatment-by-sex interaction $(p>0.1)$. In patients with high triacylglycerol levels and low HDL-cholesterol, fenofibrate reduced total cardiovascular outcomes by $30 \%(95 \% \mathrm{CI}-7 \%, 54 \%)$ in women and $24 \%(95 \%$ CI $2 \%$, $42 \%)$ in men, with no treatment-by-sex interaction $(p>0.1)$.

Conclusions/interpretation Fenofibrate improved the lipoprotein profile more in women than men. Cardiovascular event reductions with fenofibrate were consistently similar in women and men, both overall and among those with low HDLcholesterol and high triacylglycerol levels. These data provide reassurance about fenofibrate efficacy in women and men. Both sexes with type 2 diabetes should be considered for fenofibrate therapy for cardioprotection.

Keywords Adult · Cardiovascular prevention .

Cardiovascular risk · Drug effects · Fibrate · Major clinical study $\cdot$ Non-insulin-dependent diabetes mellitus $\cdot$ Randomised controlled trial $\cdot$ Sex differences $\cdot$ Statin $\cdot$ Type 2 diabetes mellitus 


\begin{tabular}{|c|c|}
\hline Abbreviations & \\
\hline ACCORD & $\begin{array}{l}\text { Action to Control Cardiovascular Risk } \\
\text { in Diabetes }\end{array}$ \\
\hline CVD & Cardiovascular disease \\
\hline FIELD & $\begin{array}{l}\text { Fenofibrate Intervention and Event } \\
\text { Lowering in Diabetes }\end{array}$ \\
\hline MI & Myocardial infarction \\
\hline NHMRC CTC & $\begin{array}{l}\text { National Health and Medical Research } \\
\text { Council Clinical Trials Centre }\end{array}$ \\
\hline
\end{tabular}

\section{Introduction}

Cardiovascular disease (CVD) is a major cause of death among women [1]. Women are more likely than men to die as a result of a first myocardial infarction (MI) or to have a second cardiovascular event [2]. In general, women have a more favourable lipid profile than men, but the differences diminish once women become postmenopausal [3]. Before the Fenofibrate Intervention and Event Lowering in Diabetes (FIELD) study [4] some [5, 6] but not all [7] fibrate trials had shown that long-term fibrate treatment reduced CVD events, although few women had been randomised into fibrate trials [5-7]. More recently the Action to Control Cardiovascular Risk in Diabetes (ACCORD) Lipid study, which evaluated fenofibrate added to background simvastatin therapy, reported a $9.1 \%$ rate of CVD events for 851 women receiving fenofibrate and a non-significantly lower rate of $6.6 \%$ in 843 women on placebo, although there was a significant interaction between treatment and sex, in favour of men $(p=0.01)$ [8]. This raised questions about the role of fenofibrate as a therapy for women with diabetes and led to a safety alert being issued by the US Food and Drug Administration $[9,10]$.

The FIELD study ( $n=9,795$, including 3,657 women) is the largest study of fibrate use in women with diabetes (International Standard Randomised Controlled Trial [ISRCTN] registration no. 64783481). In FIELD, fenofibrate did not significantly reduce the primary endpoint of CHD events (non-fatal MI and CHD death; HR 0.89; 95\% CI $0.75,1.05, p=0.16$ ) [4]. After adjustment for fenofibrate adherence and imbalanced uptake of statins and other cardiovascular drugs, this effect increased but remained non-significant (HR 0.84; 95\% CI 0.69, 1.01, $p=0.06$ ) [11]. There was no significant interaction between fenofibrate treatment and sex for total CVD events (non-fatal MI, stroke, all CVD death, and coronary and carotid revascularisation), the pre-specified endpoint for all subgroup analyses [4]. The FIELD study provides a unique opportunity to explore the effects of fenofibrate treatment by sex in more detail. This analysis also addresses the potentially adverse findings for women in the ACCORD Lipid study.

\section{Methods}

Study design and patients The study design, patient characteristics and outcome analysis of the FIELD trial have been reported [4]. In brief, 9,795 patients with type 2 diabetes aged $50-75$ years were randomised to $200 \mathrm{mg}$ micronised fenofibrate daily (Laboratoires Fournier, Dijon, France) or matching placebo. All had baseline total cholesterol levels of 3.0-6.5 mmol/1, plus a total cholesterol:HDL-cholesterol ratio $\geq 4$ or a triacylglycerol concentration of $1-5 \mathrm{mmol} / \mathrm{l}$, with no clear indication for lipid-modifying therapy at the time of study initiation. Traditional lipid levels were measured on all participants at baseline, at 4, 8 and 12 months, 2 years and at the end of the study. Apolipoproteins A-I and B were measured at baseline, 4 months, 2 years and study close. Additional cardiovascular medicines, including statins and other lipid-modifying treatments, could be commenced during the trial at the discretion of the patient's treating doctor. Medication adherence was assessed by returned tablet counts at each visit.

All patients gave written informed consent. The FIELD protocol was approved by local and national ethics committees and undertaken in accordance with the Declaration of Helsinki and Good Clinical Practice guidelines.

The primary endpoint, which was not statistically significant, [4] was the first occurrence of non-fatal MI or death from CHD. Secondary endpoints included major CVD events (the primary endpoint plus stroke and other CVD death). The prespecified outcome for all subgroup analyses, including by sex, was total CVD events (the composite of major CVD events plus coronary and carotid revascularisation). Cause-specific and total mortality were also examined [4]. In the current detailed analysis, we explore the effects of fenofibrate by sex on total CVD and the component endpoints of the total CVD outcome and also the influence of sex on the effect of fenofibrate on lipid levels measured at baseline, 4 months, 1 year, 2 years and study close. The influences of menopausal status, oestrogen use, concurrent use of statins, and metformin and insulin therapy were assessed. All lipid analyses were performed in two central laboratories with standard techniques. Rigorous quality-assurance procedures were used to verify stable assay characteristics, as previously reported [4]. Non-HDL cholesterol was calculated (total cholesterolHDL-cholesterol). The LDL-cholesterol level was calculated using standard methods [12]. Dyslipidaemia was defined a priori as fasting triacylglycerol levels $\geq 1.7 \mathrm{mmol} / \mathrm{l}$ in both sexes, and HDL-cholesterol levels $<1.03 \mathrm{mmol} / \mathrm{l}$ for men and $<1.29 \mathrm{mmol} / \mathrm{l}$ for women (according to Adult Treatment Panel [ATP] III guidelines [13]). Marked dyslipidaemia used a higher cutpoint of triacylglycerol levels $>2.3 \mathrm{mmol} / \mathrm{l}$, with the same sex-specific HDL-cholesterol cutpoints. For the purposes of this analysis, the ACCORD definition of dyslipidaemia based on the upper triacylglycerol and lower 
HDL-cholesterol tertiles, respectively (triacylglycerol $>2.3 \mathrm{mmol} / 1$ and HDL-cholesterol $\leq 0.88 \mathrm{mmol} / 1$, for both women and men) was also applied. Apolipoprotein A-I and apolipoprotein B were measured with a nephelometer (Array Protein System, Beckman-Coulter, Sydney, NSW, Australia) (Australian and New Zealand samples) or a Cobas Mira analyser (Hoffman La Roche, Basel, Switzerland) (Finland samples). The two laboratories were aligned by participation in an external quality-assurance programme coordinated by the Canadian Reference Laboratory (Vancouver, BC, Canada) using Centers for Disease Control (CDC)-traceable standard materials. Day-to-day CVs were $<5 \%$ throughout the study.

Statistical analysis All analyses were done at the National Health and Medical Research Council Clinical Trials Centre (NHMRC CTC), University of Sydney. The data were analysed on an intention-to-treat basis with SAS (version 9.2; SAS, Cary, NC, USA). The analysis of differences for baseline characteristics used $\chi^{2}$ tests for binary variables and $t$ tests for continuous outcomes unless the distribution of the data was not normal, in which case the Wilcoxon rank-sum test was used. Spearman correlations were computed on non-transformed data. Time-to-event analyses used the logrank test and the Kaplan-Meier method. HRs and 95\% CIs were generated from Cox proportional-hazards models [13]. Analyses were adjusted for covariates (including ethnicity, age, diabetes duration, BMI, waist-to-hip ratio, systolic and diastolic blood pressure, smoking, prior CVD, prior coronary revascularisation, hypertension, microvascular disease, baseline total cholesterol, LDL-cholesterol, HDL-cholesterol, creatinine, homocysteine, dyslipidaemia, microalbuminuria, macroalbuminuria, use of metformin, and use of sulfonylurea) and on-trial statin uptake [11]. ANOVA methods were used to assess the treatment-effect difference in the change in lipid and apolipoprotein levels. Heterogeneity across the sexes was assessed by determining the interaction term for sex between the treatment group and outcome, with significance drawn at two-sided $p<0.05$ with no adjustment for multiple comparisons.

\section{Results}

Baseline characteristics Women comprised $37.3 \%$ of the cohort. At the final visit, $95.0 \%$ of the women and $92.0 \%$ of the men allocated to placebo and $94.3 \%$ of the women and $91.3 \%$ of the men allocated to fenofibrate remained alive. There was no difference between women and men or between the two treatment groups in the rates of loss to follow-up $(0.3 \%$ in all groups).

Women compared with men were more likely to be younger, hypertensive and obese (Table 1). They were less likely to be current or former smokers or to have a history of CVD.
Women had lower plasma creatinine and homocysteine levels and lower albuminuria rates. They had higher levels of total cholesterol, LDL-cholesterol, HDL-cholesterol and non-HDL cholesterol. They were also more likely to be dyslipidaemic and had significantly higher baseline apolipoprotein A-I and B levels. Use of calcium antagonists, diuretics, angiotensin II receptor antagonists, and metformin (alone or in combination with other glucose-control agents) was more common in women, whereas the use of anti-thrombotic agents (including aspirin) or sulfonylureas alone was less common.

Adherence to assigned treatment and lipid drug drop-in rates At the end of the study, women and men had discontinued fenofibrate treatment at similar rates $(19.8 \%$ vs $19.3 \%$ ), equivalent to the placebo discontinuation rates. There were no statistically significant differences in adherence rates between the sexes on the basis of returned tablet counts (data not shown).

Among the participants allocated to fenofibrate, women were less likely than men to commence other active cholesterol-lowering medication, mainly statins $(16.3 \%$ vs $21 \%, p<0.001$ ), but women and men allocated to placebo had similar commencement rates of such medications (37.2\% vs $35.7 \%$ at study close, $p=0.3$ ).

Effect of fenofibrate therapy on lipid and apolipoprotein levels by sex Fenofibrate significantly reduced total cholesterol, LDL-cholesterol and triacylglycerol levels in both sexes $(p<0.001)$ (Fig. 1, electronic supplementary material [ESM] Table 1). Relative to placebo, the reductions in total cholesterol and LDL-cholesterol with fenofibrate allocation were greater in women (all $p<0.001$ ): for total cholesterol, $14.0 \%$ $(0.84 \mathrm{mmol} / \mathrm{l})$ for women vs $9.9 \%(0.49 \mathrm{mmol} / \mathrm{l})$ for men at 4 months, and $9.5 \%(0.48 \mathrm{mmol} / \mathrm{l})$ vs $5.2 \%(0.25 \mathrm{mmol} / \mathrm{l})$ at study close; and for LDL-cholesterol, $16.5 \%(0.53 \mathrm{mmol} / \mathrm{l}) \mathrm{vs}$ $9.4 \%(0.31 \mathrm{mmol} / \mathrm{l})$ at 4 months and $9.8 \%(0.29 \mathrm{mmol} / \mathrm{l})$ vs $3.3 \%(0.10 \mathrm{mmol} / \mathrm{l})$ at study close. LDL-cholesterol differences entirely accounted for the differences in cholesterol change (Fig. 1b). Fenofibrate allocation was associated with an HDL-cholesterol rise at 4 months relative to placebo in both sexes $(5.7 \%$ [0.07 $\mathrm{mmol} / \mathrm{l}]$ in women and $4.8 \%[0.05 \mathrm{mmol} / \mathrm{l}]$ in men), with the difference compared with placebo diminishing over time (Fig. 1c). Apart from an $11 \%$ greater reduction in triacylglycerol levels at 4 months in women (women $30.5 \%$ reduction [-0.6 $\mathrm{mmol} / 1]$, men $27.4 \%$ reduction $[-0.5 \mathrm{mmol} / \mathrm{l}], p=$ 0.01 ), the effect on triacylglycerol levels was similar in men and women over 5 years (Fig. 1d).

At study close, fenofibrate had almost halved the percentage of patients with dyslipidaemia, from $42.7 \%$ to $23.9 \%$ in women $(p<0.001)$ and from $34.0 \%$ to $20.2 \%$ in men $(p<0.001)$, a significantly greater reduction in women $(p=0.04)$. 
Table 1 Baseline characteristics and medication
Data are mean (SD), median (interquartile range) or $\%$

${ }^{a}$ Based on the DuBois-DuBois formula

${ }^{\mathrm{b}}$ Microalbuminuria: urine albu$\mathrm{min} /$ creatinine ratio $2.5-25 \mathrm{mg}$ / mmol for women and

$3.5-35 \mathrm{mg} / \mathrm{mmol}$ for men; and macroalbuminuria: urine albu$\mathrm{min} /$ creatinine ratio

$>25 \mathrm{mg} / \mathrm{mmol}$ for women and $>35 \mathrm{mg} / \mathrm{mmol}$ for men

${ }^{\mathrm{c}}$ Alone or with any other glucosecontrol agent

$* * * p<0.001$ for difference between women and men

\begin{tabular}{|c|c|c|}
\hline Characteristic & $\begin{array}{l}\text { Women } \\
(n=3,657)\end{array}$ & $\begin{array}{l}\text { Men } \\
(n=6,138)\end{array}$ \\
\hline Age at visit 1 , years & $61.8(6.9)$ & $62.5(6.9)^{* * *}$ \\
\hline Diabetes duration, years & $5(2-9)$ & $5(2-10)^{* * *}$ \\
\hline Weight, $\mathrm{kg}$ & $81.7(71.3-94)$ & $88.7(80-99.3)^{* * *}$ \\
\hline BMI, $\mathrm{kg} / \mathrm{m}^{2}$ & $31.5(27.8-36)$ & $29.1(26.4-32.2)^{* * *}$ \\
\hline Body surface area, $\mathrm{m}^{2 \mathrm{a}}$ & $2.1(0.2)$ & $2.3(0.2)^{* * *}$ \\
\hline \multicolumn{3}{|l|}{ Risk factors } \\
\hline Waist-to-hip ratio & $0.87(0.83-0.92)$ & $0.96(0.93-1)^{* * *}$ \\
\hline $\mathrm{HbA}_{1 \mathrm{c}}, \%$ & $6.9(6.1-7.8)$ & $6.9(6.1-7.8)$ \\
\hline $\mathrm{HbA}_{1 \mathrm{c}}, \mathrm{mmol} / 1$ & $8.4(7.1-9.8)$ & $8.4(7.1-9.8)$ \\
\hline Plasma creatinine, $\mu \mathrm{mol} / 1$ & $67.6(13.4)$ & $83.4(13.9)^{* * *}$ \\
\hline Homocysteine, $\mu \mathrm{mol} / 1$ & $8.9(7.5-10.9)$ & $9.9(8.3-11.8)^{* * *}$ \\
\hline Microalbuminuria, $\%$ b & 17.23 & $24.10^{* * *}$ \\
\hline Macroalbuminuria, $\%{ }^{\mathrm{b}}$ & 2.8 & $4.9^{* * *}$ \\
\hline Blood pressure, $\mathrm{mmHg}$ & $141 / 81$ & $140 / 83$ \\
\hline Prior CVD, \% & 18.7 & $23.6 * * *$ \\
\hline History of hypertension, $\%$ & 63.7 & $52.4^{* * *}$ \\
\hline Prior microvascular disease, $\%$ & 18.0 & $22.3 * * *$ \\
\hline Current or ex-smoker, $\%$ & 41.6 & $70.8^{* * *}$ \\
\hline \multicolumn{3}{|l|}{ Lipid and apolipoprotein variables } \\
\hline Total cholesterol, mmol/1 & $5.21(0.7)$ & $4.93(0.69)^{* * *}$ \\
\hline LDL-cholesterol, mmol/1 & $3.12(0.67)$ & $3.03(0.64)^{* * *}$ \\
\hline HDL-cholesterol, mmol/1 & $1.21(0.28)$ & $1.03(0.23)^{* * *}$ \\
\hline Non-HDL cholesterol, mmol/l & $4.01(0.70)$ & $3.90(0.67)^{* * *}$ \\
\hline Triacylglycerol, mmol/1 & $1.79(1.4-2.3)$ & $1.70(1.3-2.3)$ \\
\hline Dyslipidaemia, $\%$ & 43.1 & $34.8 * * *$ \\
\hline Apolipoprotein A-I, g/l & $1.32(1.19-1.47)$ & $1.18(1.07-1.30)^{* * *}$ \\
\hline Apolipoprotein B, g/1 & $0.99(0.18)$ & $0.96(0.17)^{* * *}$ \\
\hline \multicolumn{3}{|l|}{ Cardiovascular medication, $\%$} \\
\hline Anti-thrombotic agents & 27.2 & $33.8^{* * *}$ \\
\hline ACE inhibitors or angiotensin II receptor antagonist & 41.0 & $37.3 * * *$ \\
\hline$\beta$ blocker & 15.4 & 14.0 \\
\hline Calcium antagonist & 21.2 & $18.2 * * *$ \\
\hline Nitrate & 5.5 & 5.7 \\
\hline Diuretic agent & 22.3 & $10.9 * * *$ \\
\hline \multicolumn{3}{|l|}{ Blood-glucose-lowering medication, $\%$} \\
\hline Metformin alone & 20.6 & $15.8^{* * *}$ \\
\hline Any metformin ${ }^{\mathrm{c}}$ & 51.9 & $47.2 * * *$ \\
\hline Sulfonylurea alone & 13.7 & $18.1^{* * *}$ \\
\hline Sulfonylurea and metformin & 22.9 & 24.1 \\
\hline Insulin alone or with oral agent & 13.7 & 13.8 \\
\hline
\end{tabular}

Effect of statin therapy on fenofibrate-induced lipid changes The differences between treatment groups in total cholesterol and LDL-cholesterol in both sexes attenuated over time (ESM Fig. 1a, b) [4], but among patients not commencing statins, the effect of fenofibrate was preserved. The LDLcholesterol-lowering effect of fenofibrate was greater in these women than men at all time points: $18.1 \%$ vs $10.8 \%$ at
4 months and $20.1 \%$ vs $11.2 \%$ at study close (all $p<0.001$ ) (ESM Fig. 1). Neither statin commencement rates nor differences in body weight explained the greater LDL-cholesterol reduction in women.

Effect of insulin, metformin, and oestrogen therapy on fenofibrate-induced lipid changes There were no significant 
Fig. 1 Absolute lipid changes with fenofibrate compared with placebo from baseline to study close in women and men (measured at clinic visits at 4 months, 1 year, 2 years and study close [5 years]). (a) Total cholesterol; (b) LDL-cholesterol; (c) HDL-cholesterol; (d) triacylglycerols;

(e) apolipoprotein A-I; and (f) apolipoprotein B. The reduction in non-HDL cholesterol level by fenofibrate relative to placebo was greater in women than men at all time points. Apolipoprotein A-I and HDL-cholesterol were significantly correlated at each time point ( $r=0.45$ to $r=0.65$, all $p<0.01)$. Apolipoprotein B levels paralleled the patterns of nonHDL cholesterol $(r=0.74$ to $r=$ $0.91)$, LDL-cholesterol $(r=0.63$ to $r=0.85$ ) and triacylglycerol levels ( $r=0.25$ to $r=0.46$ ) (all $p<0.01)$. Red dashes, women, placebo; red solid line, women, fenofibrate; blue dashes, men, placebo; blue solid line, men, fenofibrate; m, months; $y$, years
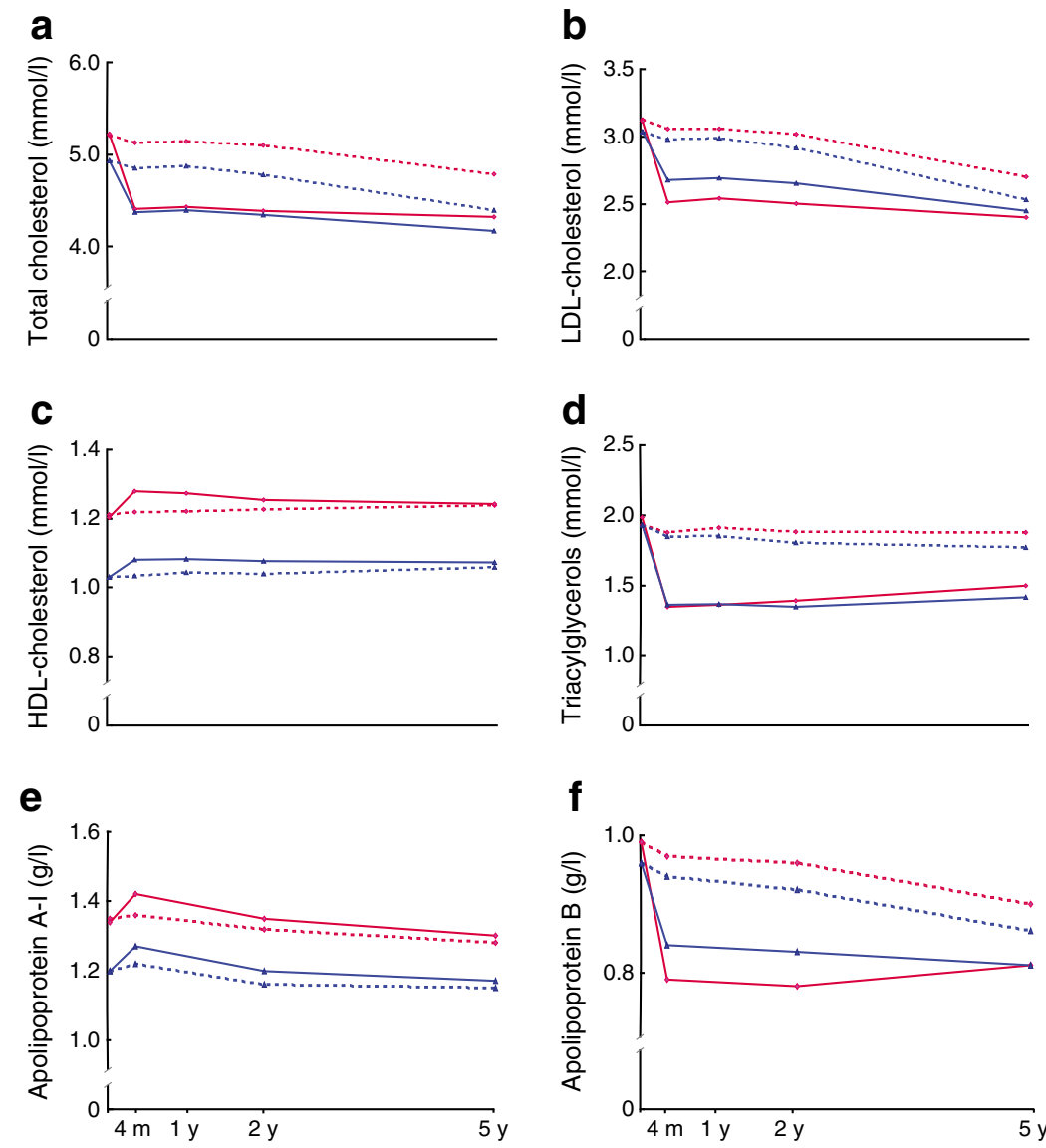

effects of initiation of metformin or insulin treatment during follow-up on the fenofibrate-induced lipid changes over 5 years. Only small numbers of women used oestrogen throughout the study, with no significant effect on lipid changes due to fenofibrate (not shown).

Effect of fenofibrate on CVD events in women The primary endpoint of the FIELD study, non-fatal MI plus death from CHD, was not significantly reduced by fenofibrate treatment (HR 0.89; 95\% CI 0.75, 1.05, $p=0.16$ ) [4]. Fenofibrate significantly reduced total CVD events, the pre-specified endpoint for all subgroup analyses, overall by $11 \%$ [4]. Total CVD events were reduced by $20 \%$ in women and nonsignificantly by $8 \%$ in men, but these sex-specific treatment effects were not significantly different (Fig. 2). In women, allocation to fenofibrate compared with placebo was associated with lower rates of non-fatal MI and revascularisation procedures, but neither was statistically significant, whereas the $22 \%$ reduction in non-fatal $\mathrm{MI}$ and the $18 \%$ reduction in revascularisation in men allocated to fenofibrate compared with placebo were statistically significant. The relative benefits of fenofibrate allocation in women compared with men did not differ statistically for any of the CVD outcomes separately or in aggregate (all $p$ values for heterogeneity $>0.1$ ) (Fig. 3).
Among patients who did not have CVD at study entry, fenofibrate reduced the risk of total CVD events by $26 \%$ ( $p=$ $0.04)$ in women and $16 \%(p=0.04)$ in men (interaction by sex, $p=0.45)$. Non-fatal MI was reduced by $41 \%$ in women $(p=0.05)$ and $29 \%$ in men $(p=0.04$; interaction $p=0.54)$. Fenofibrate reduced the risk of coronary events (CHD death

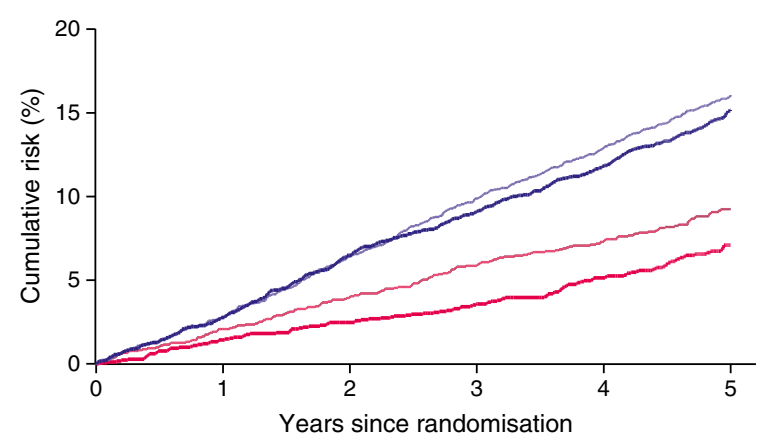

Fig. 2 Cumulative cardiovascular event rates in patients assigned to fenofibrate and placebo. Allocation to fenofibrate compared with placebo reduced total cardiovascular events (HR $0.89,95 \%$ CI $0.80,0.99, p=$ 0.035 ). For women the HR was $0.80,95 \%$ CI $0.64,0.99, p=0.04$; for men HR $0.92,95 \%$ CI $0.81,1.05, p=0.2$; sex-by-treatment interaction $p=0.3$; $p$ values are unadjusted for multiple comparisons. Lighter red, women, placebo; darker red, women, fenofibrate; lighter blue, men, placebo; darker blue, men, fenofibrate 


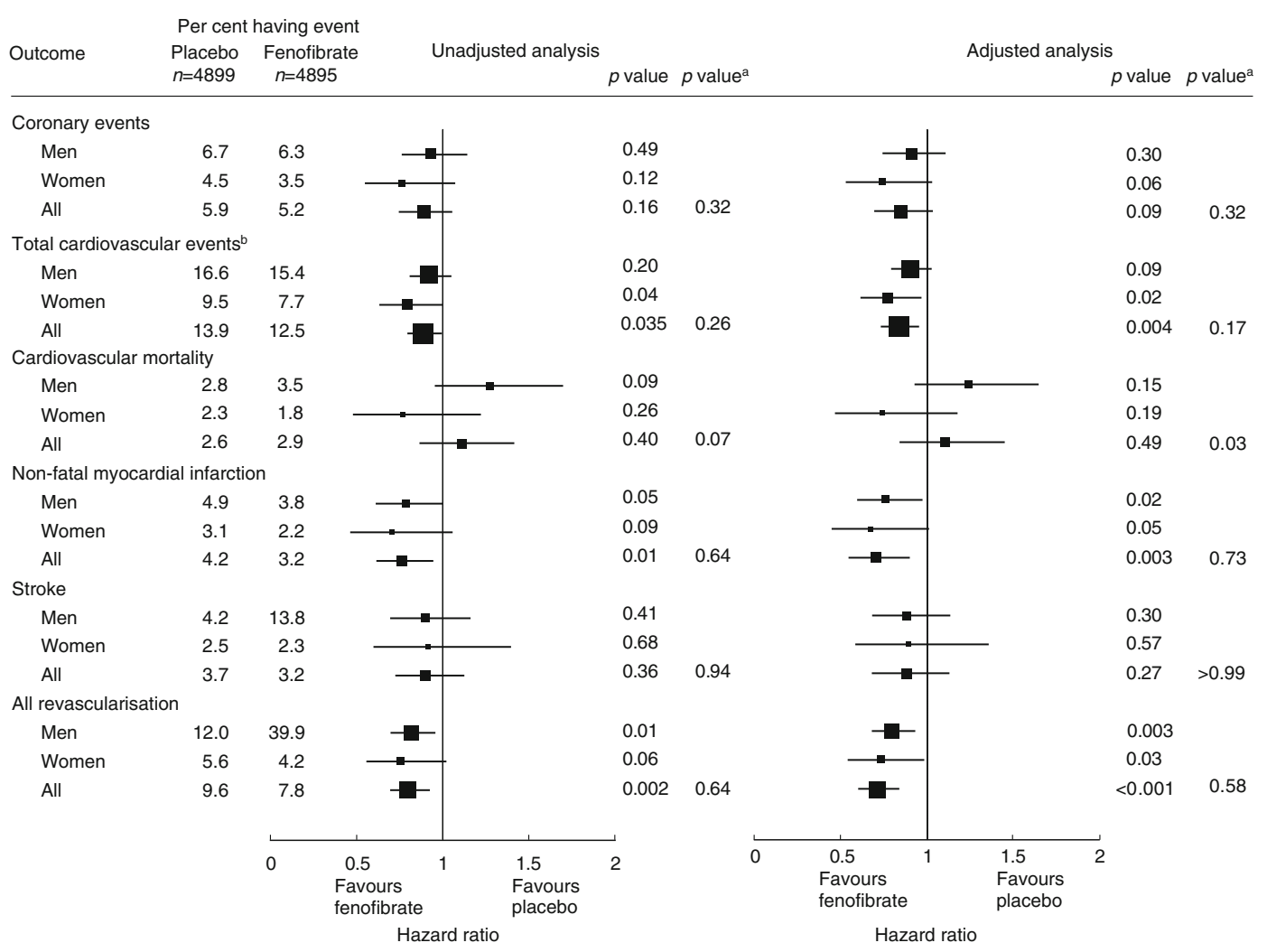

Fig. 3 HRs with 95\% CIs for the effect of fenofibrate compared with placebo for cardiovascular outcomes in 3,657 women and 6,138 men, unadjusted and adjusted for uptake of statin therapy and baseline covariates (ethnicity, age, diabetes duration, BMI, waist-to-hip ratio, systolic blood pressure, diastolic blood pressure, smoking, prior cardiovascular disease, prior coronary revascularisation, hypertension, microvascular

or non-fatal MI, the primary study endpoint) by $41 \%$ ( $p=$ $0.02)$ in women and $17 \%$ in men $(p=0.17)$ and revascularisation by $31 \%$ in women $(p=0.06)$ and $27 \%(p=$ $0.002)$ in men. Again, interactions between sex and treatment were not statistically significant, as was also the case among those patients with dyslipidaemia (all interaction $p$ values $>$ 0.24). For example, the effects of fenofibrate on total CVD events among those with dyslipidaemia were $24 \%$ (95\% CI $-4 \%, 45 \%)$ reduction in women and $10 \%(95 \% \mathrm{CI}-9 \%, 26 \%)$ reduction in men, and among those with marked dyslipidaemia $(95 \% \mathrm{CI}-7 \%, 54 \%)$ reduction in women and $24 \%$ (95\% CI $2 \%, 42 \%$ ) in men [14]. Applying the ACCORD Lipid definition of dyslipidaemia [8] gave similar results (ESM Table 2).

Adjusted effects of fenofibrate treatment With adjustment for statin use and other baseline covariates, [11] allocation to fenofibrate reduced total CVD events by $17 \%$ overall $(95 \%$ CI $6 \%, 27 \%$, and in women by $30 \%(95 \%$ CI $8 \%, 46 \%)$ although not significantly in men (13\%; $95 \%$ CI $-1 \%, 24 \%)$, with no statistical evidence of heterogeneity of effect by sex ( $p=0.17)$. There was no significant reduction in non-fatal MI among women $(35 \% ; 95 \% \mathrm{CI}-5 \%, 61 \%)$ but there was for men $(28 \%$; $95 \%$ CI $5 \%, 47 \%)$. Revascularisation procedures

disease, baseline total cholesterol, LDL-cholesterol, HDL-cholesterol, creatinine, homocysteine, dyslipidaemia, microalbuminuria, macroalbuminuria, use of metformin and use of sulfonylurea). ${ }^{\mathrm{a}} p$ for heterogeneity between men and women. ${ }^{\mathrm{b}}$ Cardiovascular events was the pre-specified outcome for subgroups

were reduced by $34 \%(95 \%$ CI $7 \%, 55 \%)$ in women and by $27 \%$ (95\% CI 13\%, 40\%) for men (Fig. 3).

Safety of fenofibrate in women There were no significant differences in total cancer incidence with allocation to fenofibrate in either sex (ESM Table 3). Nor was there any excess of rhabdomyolysis, irrespective of statin commencement during follow-up in either sex. Small excesses of pancreatitis and pulmonary embolism with fenofibrate did not differ significantly by sex. End-stage renal disease requiring dialysis was not increased with treatment in men or women.

\section{Discussion}

On the basis of ACCORD Lipid study results [8], the Food and Drug Administration reviewed the cardiovascular safety of fenofibrate in women with diabetes [9]. Our results indicate that fenofibrate is generally safe for women with type 2 diabetes. Although in the FIELD study, the primary endpoint of non-fatal MI plus coronary death was not significantly reduced, the secondary endpoint of total CVD events was 
reduced (without adjustment for multiple comparisons). Women allocated fenofibrate, including those with dyslipidaemia at baseline, had risk reductions in CVD events that did not differ statistically significantly from those in men, and for some event types tended to have greater benefit. Women also had significantly greater improvements in traditional lipids variables and in apolipoprotein B levels.

Differences between treatment groups in LDL-cholesterol concentrations always remained larger in women, and were most clearly evident in statin-naive patients. These differences were not explained by differences in baseline characteristics such as body habitus, insulin or metformin therapy, menopausal status or oestrogen use. The apparent attenuation over time of the effect of fenofibrate on lipid and apolipoprotein levels in both sexes combined was driven by the higher rate of statin initiation in the placebo groups than in those allocated to fenofibrate.

The significant differences between women and men in the effects of fenofibrate on lipid and apolipoproteins were unexpected. Women were lighter than men, with a smaller body surface area, but had a higher body mass index (BMI, $31.5 \mathrm{vs}$ $29.1 \mathrm{~kg} / \mathrm{m}^{2}$ ) (Table 1). Sex differences in mass, BMI and body surface area might be associated with differences in the volume of distribution or other pharmacodynamic properties of fenofibrate. Oestrogenic hormones are potential confounding factors, given that they are known to increase HDLcholesterol and reduce LDL-cholesterol $[15,16]$ and to influence lipoprotein-related enzyme levels. In our study, most women $(94 \%)$ were postmenopausal (average age at study entry 61 years), but the effects of fenofibrate in premenopausal and postmenopausal women were not different for lipoprotein measures or for clinical CVD event reductions, and too few women continued exogenous oestrogen therapy to have any plausible impact on the results. Nor did insulin use or differences in statin uptake between the sexes explain the difference in LDL-cholesterol and fenofibrate response (see ESM Fig. 1 and Simes et al [11]). Uptake of statin therapy during the trial may have confounded the treatment effects. We adjusted the results for statin uptake (and covariates), which is considered a better method of accounting for this than using analytical methods that do not maintain the randomised comparisons [11]. In fact, there was little difference between the unadjusted results and the results adjusted for statin use and covariates. Metformin use, which can improve the lipid profile [17], was more common in women, but in FIELD, metformin use did not contribute materially to the observed sex differences in lipid and apolipoprotein changes.

Previous studies with fenofibrate or, indeed, any fibrate have not reported a sex difference in responsiveness to therapy. However, most of the large prospective CVD intervention studies with fibrate monotherapy have either not included women or have had relatively small numbers of women $[5$, 6], and even fewer have had women with diabetes.
The ACCORD Lipid study had 1,694 women (31\% of patients) randomly assigned to fenofibrate or placebo on background statin therapy [8]. Lipid changes by sex in ACCORD Lipid have yet to be published in a journal article, but have been reported to the US Food and Drug Administration [9] and show larger LDL-cholesterol reductions in women. In contrast to our study, ACCORD Lipid reported that the primary outcome (CVD death or non-fatal MI or non-fatal stroke) was less frequent $(11.2 \%)$ in men using the combination of fenofibrate and simvastatin than in men receiving simvastatin alone $(13.3 \%)$, but the rate for women was non-significantly more frequent in the combination therapy group $(9.1 \%$ vs $6.6 \%)$; the difference between the sexes was significant ( $p=0.01$ for interaction) [18]. Such sexspecific differences in treatment benefits were not apparent among participants with dyslipidaemia in ACCORD Lipid [18], where CVD benefits were greatest [8]. FIELD has also reported similarly large CVD benefits in men and women with diabetes and dyslipidaemia [19]. Only a small number of women in FIELD met the ACCORD Lipid definition for dyslipidaemia, but patterns of fenofibrate effect among them were similar to those for men (ESM Table 2).

There are several potential reasons for the discrepant results between FIELD and ACCORD Lipid. All patients in ACCORD Lipid but fewer than one-third of FIELD patients received statin therapy. Patients in FIELD may have had a higher background risk by virtue of not being on statin therapy at randomisation, but they had a shorter duration of diabetes and a lower $\mathrm{HbA}_{1 \mathrm{c}}$ at study entry. Nevertheless, CVD event rates among women in the control arm in FIELD appeared to be about $50 \%$ higher than in ACCORD Lipid (ESM Table 2). The apparent higher event rate combined with the greater number would offer substantially more power in FIELD to evaluate the effects of fenofibrate in women. It is possible that the non-significantly adverse CVD result for women in ACCORD Lipid was just the play of chance. It is important to note that event rates were reduced similarly in dislipidaemic women and men in ACCORD Lipid [19]. The results do suggest that the combination of fenofibrate with a statin will be most beneficial for CVD prevention in patients with dyslipidaemia on statin therapy and are consistent with findings in FIELD that the effect is greatest in patients with dyslipidaemia [19].

\section{Conclusion}

In summary, the FIELD study showed that both men and women with diabetes had significantly improved lipoproteins with fenofibrate therapy, with changes in women being greater than in men. The primary endpoint of the study was not 
statistically significantly reduced, but total CVD events were significantly lowered with allocation to fenofibrate. In prespecified analyses by sex, there was no evidence that women did worse than men and for several endpoints, including total CVD events, there was a trend suggesting that women may have benefited more. These benefits were particularly large in those with dyslipidaemia, consistent with the findings in ACCORD Lipid. These results suggest that fenofibrate is effective for improving an adverse lipoprotein profile and for reducing total CVD event risk in women with type 2 diabetes, especially those with dyslipidaemia.

Acknowledgements The authors had full access to all study data. Data were analysed by statisticians D. Zannino and K. Mann at the NHMRC CTC, University of Sydney. We thank R. Pike for assistance with the manuscript and M. Donoghoe for assistance with analyses (both from NHMRC CTC).

Funding FIELD was supported by a grant from Laboratoires Fournier, Dijon, France (now part of Abbott Pharmaceuticals) and by the National Health and Medical Research Council of Australia (grants 457103, 1024105 and 1037786) and was coordinated independently by the NHMRC CTC.

Duality of interest ACK and MCDE have received speaker's bureau fees from Solvay (now Abbott Pharmaceuticals). ACK and AJJ have received travel and/or research grant support from Solvay (now Abbott Pharmaceuticals). All other authors declare that there is no duality of interest associated with their contribution to this manuscript.

Contribution statement $\mathrm{MCDE}$, JDB and ACK designed the study; MCDE, AJJ and ACK wrote the manuscript; LL researched the data; DZ and KPM analysed the data; MCDE, AJJ, LL, DZ, KPM, JDB, BGAS, KP, JS and ACK interpreted the data; LL, DZ, KPM, JDB, BGAS, KP and JS contributed to the discussion and reviewed and revised the manuscript. All authors approved the final manuscript. ACK is the guarantor of this work.

\section{References}

1. World Health Organization (2008) World health statistics 2008. World Health Organization, Geneva, pp 1-110

2. Vaccarino V, Parsons L, Every NR, Barron HV, Krumholz HM (1999) Sex-based differences in early mortality after myocardial infarction. National Registry of Myocardial Infarction 2 Participants. N Engl J Med 341:217-225

3. Wang X, Magkos F, Mittendorfer B (2011) Sex differences in lipid and lipoprotein metabolism: it's not just about sex hormones. J Clin Endocrinol Metab 96:885-893

4. FIELD Study Investigators, Keech A, Simes RJ, Barter P et al (2005) Effects of long-term fenofibrate therapy on cardiovascular events in 9795 people with type 2 diabetes mellitus (the FIELD study): randomised controlled trial. Lancet 366:1849-1861
5. Rubins HB, Robins SJ, Collins D et al (1999) Gemfibrozil for the secondary prevention of coronary heart disease in men with low levels of high-density lipoprotein cholesterol. Veterans Affairs High-Density Lipoprotein Cholesterol Intervention Trial Study Group. N Engl J Med 341:410-418

6. Frick MH, Elo O, Haapa K et al (1987) Helsinki Heart Study: primary-prevention trial with gemfibrozil in middle-aged men with dyslipidemia. Safety of treatment, changes in risk factors, and incidence of coronary heart disease. N Engl J Med 317: $1237-1245$

7. BIP Study Group (2000) Secondary prevention by raising HDL cholesterol and reducing triglycerides in patients with coronary artery disease: the Bezafibrate Infarction Prevention (BIP) study. Circulation 102:21-27

8. ACCORD Study Group (2010) Effects of combination lipid therapy in type 2 diabetes mellitus. N Engl J Med 362:1563-1574

9. US Food and Drug Administration (2011) FDA drug safety communication: review update of Trilipix (fenofibric acid) and the ACCORD Lipid trial Silver Spring, MD: US FDA (updated 8 Dec 2011). Available from www.fda.gov/Drugs/DrugSafety/ucm 278837. htm\#sa, accessed 10 Jul 2014

10. Nilsson PM (2010) ACCORD and risk-factor control in type 2 diabetes. N Engl J Med 362:1628-1630

11. Simes J, Voysey M, O'Connell R et al (2010) A novel method to adjust efficacy estimates for uptake of other active treatments in longterm clinical trials. PLoS One 5:e8580

12. Friedewald W, Levy R, Fredrickson D (1972) Estimation of the concentration of low-density lipoprotein cholesterol in plasma, without use of the preparative ultracentrifuge. Clin Chem 18:499-502

13. Expert Panel on Detection, Evaluation, and Treatment of High Blood Cholesterol in Adults (Adult Treatment Panel III) (2001) Executive summary of the Third Report of The National Cholesterol Education Program (NCEP) Expert Panel on Detection, Evaluation, and Treatment of High Blood Cholesterol in Adults (Adult Treatment Panel III). JAMA 285:2486-2497

14. Collett D (2003) Modelling survival data in medical research, 2nd edn. Chapman \& Hall, London

15. Krauss RM, Lindgren FT, Wingerd J, Bradley DD, Ramcharan S (1978) Effect of estrogens and progestins on high density lipoproteins. Lipids 14:113-118

16. Campos H, Walsh BW, Judge H, Sacks FM (1997) Effect of estrogen on very low density lipoprotein and low density lipoprotein subclass metabolism in postmenopausal women. J Clin Endocrinol Metab 82: 3955-3963

17. Wulffelé MG, Kooy A, de Zeeuw D, Stehouwer CDA, Gansevoort RT (2004) The effect of metformin on blood pressure, plasma cholesterol and triglycerides in type 2 diabetes mellitus: a systematic review. J Intern Med 256:1-14

18. Ginsberg HN (2011) Dyslipidemia in diabetes. The ACCORD Lipid Trial [slide set]. Loss of gender difference in primary outcome in the presence of dyslipidemia. p. 58. Silver Spring: US Food and Drug Administration. Available from www.fda.gov/downloads/ AdvisoryCommittees/CommitteesMeetingMaterials/Drugs/ EndocrinologicandMetabolicDrugs AdvisoryCommittee/ UCM258121.pdf, accessed 10 Jul 2014

19. Scott R, O'Brien R, Fulcher G et al (2009) The effects of fenofibrate treatment on cardiovascular disease risk in 9795 people with type 2 diabetes and various components of the metabolic syndrome: the FIELD study. Diabetes Care 32:493-498 